\title{
Os princípios da Teoria de Sistema de Atividades na análise do processo de construção de atividades pedagógicas com emprego de dispositivos móveis no ensino de conhecimentos Químicos
}

Principles of Activity Theory in analysing the process of construction of pedagogic activities with the use of mobile devices in the Chemistry learning

Liliane da Silva Coelho Jacon ${ }^{1}$ Irene Cristina de Mello ${ }^{2}$

\section{Resumo}

Os dispositivos móveis despontam-se como os protagonistas na garantia de um recurso favorável à conexão, minimizando sobremaneira as limitações espaço-temporais das pessoas e possibilitando o emprego emergente da aprendizagem com mobilidade ( $m$ learning). O uso de dispositivos móveis na práxis pedagógica implica na necessidade de uma maior aproximação dos professores em formação inicial e seus formadores no sentido de viabilizar que ocorra a incorporação desta tecnologia móvel nos cursos de Licenciatura. Esta "aproximação" significa a viabilização de encontros para discutir, refletir e dialogar sobre a incorporação desta tecnologia no processo de ensino-aprendizagem. Nesta pesquisa, duas educadoras, uma delas formadora de professores de Química e a outra, professora pesquisadora de informática e educação, realizaram alguns encontros para discutir e refletir sobre o emprego desta tecnologia móvel na licenciatura. A opção metodológica fundamenta-se na abordagem qualitativa com elementos de pesquisa-ação tendo como base os pressupostos teóricos da Teoria dos Sistemas de Atividade (ENGESTRÖM, 1999). Portanto, o estudo situado no campo dos debates sobre o emprego de dispositivos móveis no ensino de Química foi desenvolvido no âmbito do curso de Licenciatura em Química da Universidade Federal de Rondônia. Dentre um conjunto de sistemas de atividade, em que atuaram discentes e docentes, com seus objetos de atividade

\footnotetext{
${ }^{1}$ Universidade Federal de Rondônia - UNIR | liliane285@gmail.com

2 Universidade Federal do Mato Grosso - UFMT | icmello@terra.com.br
} 
específicos, é apresentado o Sistema de atividade relacionado à construção dessas atividades. A análise do SA sob a perspectiva dos cinco princípios da Teoria de Sistema de Atividade indicam que o processo de participação colaborativa dos encontros, da aplicação das atividades junto aos discentes do curso de licenciatura e a elaboração de artigos científicos demonstrou a evolução qualitativa do professor formador de química.

Palavras-chave: Formação continuada de formadores professores, teoria de sistema de atividade, Dispositivos móveis, tecnologias de informação e comunicação, mobilidade.

\section{Abstract}

Mobile devices emerge as the major players to ensure a favorable resource to connect, minimizing the limitation space-time constraints among people and enabling the use of emerging mobile learning (m-learning). The use of mobile devices in pedagogic praxis implies in a closer link between teachers in their initial development and their teacher educator in order to enable the incorporation of this mobile technology in undergraduate courses. This "approach" means the facilitation of meeting to discuss, reflect and talk about the incorporation of this technology in the teaching learning process. In this research, two professors had meetings to discuss and reflect about the employment of this mobile technology in the undergraduate course. One of them, a Chemistry teacher educator and the other is a computer and education teacher-research. The methodological approach is based on a qualitative method with some elements of action-research based on theoretical assumptions of the Activity Theory (ENGESTRÖM, 1999). Therefore, the study based on the debates over the use of mobile devices in the teaching of chemistry was developed as part of the undergraduate course in Chemistry at the Federal University of Rondonia. Among a set of activities, in which students and professors were present with their objects of specific activities, was presented the Activity system related to the construction of those activities. The analysis of SA from the perspective of the 5 principles of Activity Theory points out that the process of collaborative participation in the meetings, the implementation of activities with the students of the degree course and the preparation of scientific papers demonstrated the qualitative evolution of the chemistry teacher educator.

Keywords: Continuing education of teacher educators, Activity Theory, mobile device systems, information and communication technology, mobility. 


\section{Introdução}

Incentivadas por sofisticadas inovações nos meios tecnológicos de informação e comunicação, é preciso admitir a inegável transformação que a Internet e seus aparatos trouxeram para as relações sociais, com possibilidades ampliadas e, sobretudo, com mais facilidades no acesso à informação. A conexão, muito além da rede mundial de computadores, passou a ser entendida como uma rede entre pessoas. E, neste cenário, os dispositivos móveis despontam-se como os protagonistas na garantia de um recurso favorável à conexão, minimizando sobremaneira as limitações espaço-temporais dos sujeitos, que oportuniza o emprego emergente da aprendizagem com mobilidade $(\mathrm{m}$ learning). Aprender com mobilidade, amplia os espaços-tempos de aprendizagem em que as pessoas aproveitam os mais diferentes locais, a qualquer horário, para desenvolver atividades que envolvam aprendizagem (SACCOL et al, 2011,p.14).

Repensar a educação a partir dessa realidade, e dos desafios que ela traz consigo, requer a compreensão da função docente diante das possibilidades oferecidas pelos dispositivos móveis no contexto escolar. Para tanto, a formação inicial docente necessita integrar processos de reforma educacional que resultem em verdadeira modificação das práticas dos formadores na preparação dos futuros professores, visando incorporar essa tecnologia móvel. A aproximação entre professor e tecnologia deve ocorrer, de preferência, nas licenciaturas e nos cursos de pedagogia. Desta forma, surge um questionamento: Como um formador de professores pode auxiliar seus alunos (professores em formação inicial) a empregar pedagogicamente essa tecnologia de conexão à rede sem fio e o uso de dispositivos móveis em sua prática escolar? Para alterar as práticas existentes, os professores necessitam de apoio nas áreas em que se almeja a mudança através de ações de desenvolvimento profissional.

Nesta pesquisa, uma formadora de professores aceitou o desafio em participar de encontros para discutir e elaborar atividades didático-pedagógicas com emprego de dispositivos móveis no ensino de conhecimentos químicos junto aos seus alunos no curso de Licenciatura em Química da Universidade Federal de Rondônia. Estas ações e atividades foram realizadas através de uma perspectiva dialógica e colaborativa em parceria com uma pesquisadora de Informática e Educação baseada nos pressupostos da Teoria de Sistema de Atividade (ENGESTRÖM, 1999). Ao reconhecer os princípios da TSA não só como uma possibilidade teórica, mas também metodológica; ela foi utilizada para a análise do SA do processo de construção de atividades pedagógicas para o ensino de conhecimentos químicos com emprego dos dispositivos móveis. Portanto, o objetivo desta pesquisa é apresentar e discutir este SA.

\section{Fundamentação teórica}

A abordagem teórica deste estudo é constituída de elementos da teoria da atividade (Leontiev) que foram desdobradas a partir das ideias de Vygotsky e que, posteriormente, foram discutidas por Engeström (1999) e outros.

Atividades são "processos psicologicamente caracterizados por aquilo a que o processo, como um todo, se dirige (seu objeto), coincidindo sempre com o objetivo que estimula o sujeito a executar esta atividade, isto é, o motivo" (LEONTIEV, 2001, p.68). Um sujeito encontra-se em atividade quando o objetivo de sua ação coincide com o motivo de 
sua atividade. Para ele, a atividade envolve a elaboração das noções de objeto e meta, sendo de importância fundamental o objeto para uma análise da motivação. A principal coisa que distingue uma atividade de outra é a diferença de seus objetos. O objeto, em mudança e em desenvolvimento, de uma atividade relaciona-se a um motivo que a impulsiona.

O trabalho de Leontiev estabeleceu a ideia de que as diferentes atividades são distinguidas por seus objetos e que é a transformação do objeto/meta que leva à integração dos elementos do sistema de atividade (DANIELS, 2003, 114).

Para Engeström, a atividade é formação coletiva, sistêmica, com uma estrutura mediacional complexa. Um sistema de atividade produz ações e é realizado por meio de ações. A atividade, porém, não se reduz as ações, que tem vida relativamente curta, e um começo e fim temporalmente bem definidos(DANIELS, 2003, p.115)

Camillo e Mattos (2014) discutem que as necessidades humanas podem ser de domínio biológico (por exemplo: saciar a fome) ou ser uma necessidade cultural (por ex. a utilização de uma nova ferramenta para a resolução de um determinado problema). Estes pesquisadores, ao citarem Engeström, afirmam que a dinâmica da atividade se dá pelas contínuas contradições internas e externas a ela que se fazem presentes. As necessidades humanas, ou seja, aquilo que estrutura a atividade, acaba por estar sintetizado no objeto desta atividade. Daí a atividade ser orientada por objetos (p.132).

A mediação é um conceito fundamental da Teoria da Atividade e a primeira geração é baseada nesta concepção vygotskiana, representada pelo triangulo equilátero - em que nos vértices tem-se $\mathrm{O}$ sujeito, $\mathrm{O}$ objeto $\mathrm{e}$ artefatos mediacionais (ferramentas $\mathrm{e}$ signos/linguagens). Engeström (1999) expandiu esta representação original triangular, pois seu propósito era "possibilitar um exame dos sistemas de atividade no nível macro do coletivo e da comunidade, em preferência a um nível micro de concentração no agente individual operando com ferramentas" (DANIELS, 2003, p.118). A Figura 1 ilustra este modelo.

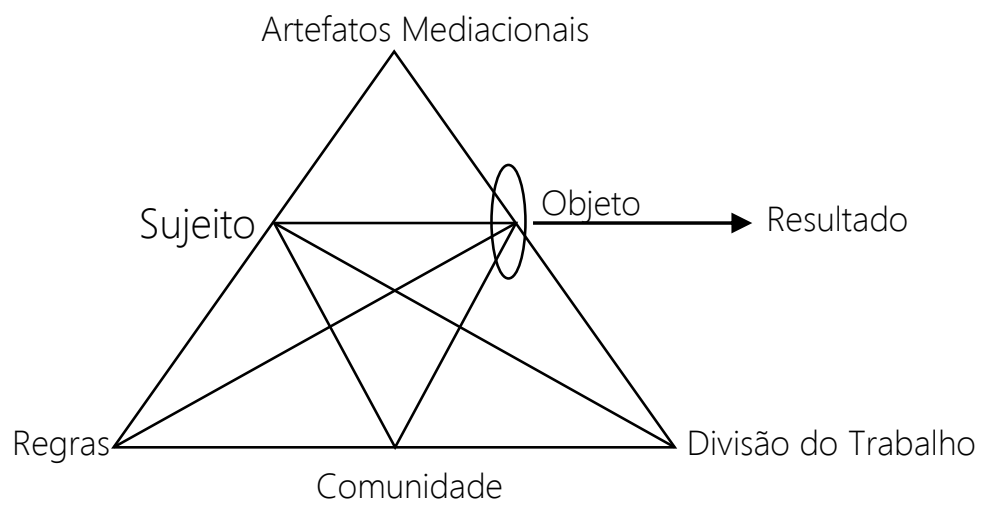

Figura 1 - Esquema representativo dos elementos de um Sistema de Atividade Fonte: a partir de Daniels (2003)

Ao incorporar as estruturas sociais que agem, elas mesmas, para organizar e restringir a própria atividade, Engeström (1999) mudou o foco. Nesta segunda geração, busca-se representar os elementos coletivos pela adição dos elementos comunidade, regras e divisão do trabalho. 
Camillo e Mattos (2014) citam o exemplo da atividade de caça realizada por um grupo humano primitivo, para exemplificar que "enquanto na atividade animal existe uma fusão entre motivo (o que leva a fazer) e objeto (para o que se dirige), na atividade humana a divisão social do trabalho dá sentido às ações individuais e proporciona a separação entre objeto e motivo" (p.132). A crítica que emergiu desta segunda geração se deve a insensibilidade à diversidade cultural e diálogo entre diferentes tradições, culminando na terceira geração da Teoria da Atividade.

Engeström recorreu a ideias de dialogicalidade e multivocalidade a fim de expandir a estrutura da $2^{a}$.geração e, ao elaborar a $3^{a}$.geração desta teoria, sua intenção foi "desenvolver ferramentas conceituais para compreender os diálogos, as múltiplas perspectivas e redes dos sistemas de atividade interativa" (p.121). Para ele, a atividade é "alcançada pela negociação, pela orquestração e pela luta constante entre diferentes metas e perspectivas de seus participantes" (p.119)

A construção de objetos mediada por artefatos [...] é um processo colaborativo e dialógico, em que diferentes perspectivas [...] e vozes [...] se encontram, colidem e fundem. As diferentes perspectivas estão enraizadas em diferentes comunidades e práticas, que continuam a coexistir no mesmíssimo sistema de atividade coletivo (ENGESTRÖM, 1999, p.382 apud DANIELS, 2003, p.120)

Engeström (2003) afirma ser importante "ir além do sistema de atividade singular e examinar e trabalhar para a transformação de redes de atividades" (DANIELS, 2003, p.122). Importante observar que o modelo teórico de Engeström é o fato de os Sistemas de Atividades interagirem e formarem redes, não sendo possível estudá-los isoladamente. Ele se interessou pelo processo de transformação social e, em razão disto, leva em conta a natureza conflituosa da prática social; analisando a "instabilidade (tensões internas) e a contradição como a força motriz da mudança e do desenvolvimento" para concluir que não é apenas o sujeito, mas o ambiente, que é modificado pela atividade mediada (DANIELS, 2003, p.120). Segundo ele, sua pesquisa é baseada na intervenção desenvolvimental, e tem uma relação dialética, dialógica com a atividade, ao enfocar as contradições como causativas e as perturbações como indicadoras de potencial (p.123).

A Teoria do Sistema de Atividade pode ser resumida em cinco princípios, segundo Daniels (2003,p.123-125): No primeiro princípio assume-se que a unidade primária de análise é todo um sistema de atividade coletivo, mediado por artefato e orientado a objeto, visto em suas relações de rede com outros sistemas de atividade. É preciso compreender que ações individuais ou grupais voltadas para um objetivo compõem unidades independentes, mas subordinadas ao analisar-se a rede, o sistema de atividade inteiro.

O segundo princípio invoca a multivocalidade. As múltiplas vozes em uma comunidade com divisão de trabalhos distintos criam posições/pontos de vista/interesses diferentes, exigindo ações de tradução e negociação entre seus participantes. A historicidade é o terceiro princípio: longos períodos de tempo mudam e transformam o sistema de atividade inicialmente analisado, que só pode ser compreendido com base em sua própria história, no local da atividade e de seus objetos; e também como história de ideias teóricas e ferramentas que moldaram a atividade.

As contradições como fontes de mudança e desenvolvimento compõe o quarto princípio. As tensões e perturbações ocorrem quando um sistema de atividade adota, por exemplo, um novo elemento de fora (uma nova tecnologia, um novo objeto), causando 
contradições em que algum elemento antigo (por exemplo as regras ou a divisão do trabalho) colide com o novo.

O quinto princípio "proclama a possibilidade de transformações expansivas nos sistemas de atividade".Para Engeström (1999)

\begin{abstract}
Uma transformação expansiva é realizada quando o objeto e o motivo da atividade são reconceituados para abraçar um horizonte mais amplo de possibilidades [...] Um ciclo total de transformação expansiva pode ser compreendido como uma jornada coletiva pela zona de desenvolvimento proximal (Engeström, 1999 apud Daniels, 2003, p.125)
\end{abstract}

\title{
Metodologia
}

Fundamentada nestas ideias e ao fazer uma reflexão acerca dos conceitos instituídos na Teoria de Sistema da Atividade de Engeström (1999), utilizou-se desta como estratégia metodológica para analisar o processo de encontros para discutir e construir atividades pedagógicas com emprego dos dispositivos móveis no ensino de conhecimentos químicos.

Segundo os pressupostos teóricos da Teoria da atividade, o sujeito aprende em atividade, esta mediada por artefatos. Os artefatos mediacionais neste estudo foram: os diálogos com heterogeneidade de linguagens entre as professoras (química, informática e educacional); o emprego dos dispositivos móveis no ensino de conhecimentos químicos; e dos novos conceitos/conhecimentos decorrente das leituras de artigos científicos sobre mobilidade e incorporação desta tecnologia na Educação.

Já a Teoria dos Sistemas de Atividade (TSA) postula mais três elementos que influenciam a atividade do sujeito: a comunidade que ele está inserido, a divisão do trabalho e as regras a que ele está submetido nessa comunidade (vide Figura 2). A comunidade corresponde a todo grupo acadêmico participante do estudo e, também, aqueles que indiretamente estão interligados ao SA, ou seja, os demais docentes dos departamentos de Química e Informática, do grupo interdisciplinar EDUCIENCIA da universidade, diretores de núcleo, orientador de doutorado etc.

A principal regra para construir as atividades foram utilizar os conceitos de mobilidade ( $m$-learning), ou seja, em como ampliar os espaços-tempos da sala de aula de forma que os discentes (professores em formação inicial) aproveitassem os mais diferentes locais, a qualquer horário, para realizar seus estudos de conhecimentos químicos (JACON et.al, 2014a, 2014b). Outra regra foi buscar desenvolver atividades com uso de recursos hipermidiáticos, ou seja, em que as ideias e os pensamentos pudessem ser expressos em formatos/mídias distintas, tais como texto, imagem, animação, música, voz, ilustrações através de montagens de conexões em rede (links). (JACON et al, 2013).

O processo de construção de atividades pedagógicas e, posteriormente, a elaboração de artigos científicos, compreendem um conjunto de ações e operações realizados numa perspectiva dialogada e de forma colaborativa, entre a pesquisadora e a formadora de professores de química. Foram realizados dez encontros, entre os dias 3 de dezembro de 2012 e 7 de maio de 2013, com duração média de uma hora cada. A divisão de trabalhos pode ser resumida entre nas seguintes ações: Leitura de artigos científicos; manuseio para descobrir modos de interação com os dispositivos móveis (tablet e smartphone); levantamento das características de hardware e de software (aplicativos); escolha de assunto/tema de química e de estratégia a ser adotada na atividade pedagógica; levantamento de aplicativos da área Química; elaboração de artigos científicos e avaliação 
da prática de ensino utilizando dispositivos móveis com professores em formação inicial de Química. A única ação/operação que ficou exclusivamente sob a responsabilidade da pesquisadora foi o desenvolvimento do aplicativo (programação), embora as ideias para construção do aplicativo tenha sido realizados de forma compartilhada.

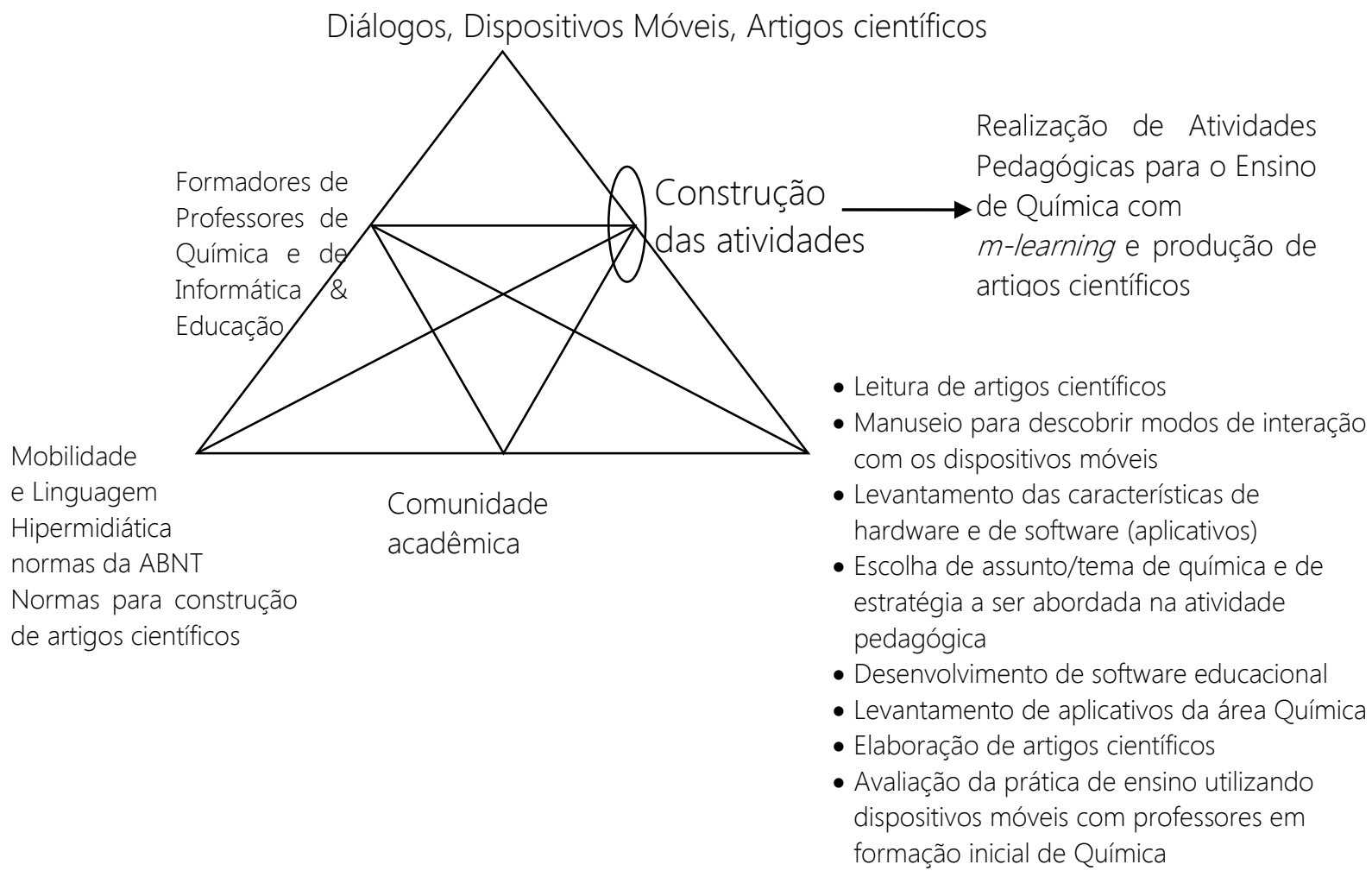

Figura 2 - Esquema representativo do SA para construção de atividades pedagógicas com o emprego dos dispositivos móveis. Fonte: autora a partir de Engeström (1999)

Nesta pesquisa, são inúmeros os SA constituintes de acordo com o primeiro princípio da TSA. Como prescreve a TSA, os inúmeros SA que constituem processos complexos de atividade humana, como num curso de Licenciatura em Química com sua comunidade acadêmica de docentes e discentes numa universidade da região norte do Brasil. Portanto, há de se considerado, nesta rede outros tantos SA possíveis constituintes, tais como: as atividade do grupo interdisciplinar de ensino de Ciências (EDUCIENCIA), as atividades dos discentes do curso de licenciatura em química, as atividades da pesquisadora de Informática e Educação enquanto doutoranda em Educação em Ciências e professora do departamento de Ciência da Computação, as atividades da Formadora de Professores do curso de Licenciatura em Química enquanto doutoranda em Educação e professora responsável pelas disciplinas de Estágio Supervisionado e responsável pelo projeto pedagógico do curso etc. Há ainda outros importantes vínculos entre as SA por meio de divisão de trabalho e das regras de funcionamento de cada delas a fim de compreender também a comunidade e a cultura acadêmica da universidade, o projeto pedagógico e a matriz curricular do curso, as regras acadêmicas, os horários, etc.

Ao analisar o SA do processo de construção de atividades pedagógicas, identifica-se o segundo princípio: a multivocalidade. Nele, pode-se observar as múltiplas vozes que povoam o SA analisado: os interesses da pesquisadora de Informática e Educação, os 
pontos de vista divergentes e congruentes da Formadora de Professores de Química, as opiniões dos discentes do curso de licenciatura durante a aplicação das atividades de $m$ learning, as sugestões do grupo interdisciplinar de Ciências da Universidade (EDUCIENCIA), as sugestões da orientação de doutorado.

Ao invocar a multivocalidade, é preciso destacar o artefato mediacional dos diálogos com heterogeneidade de linguagens envolvidas. As construções enunciativas de cada educadora estão relacionadas à formação de uma área de conhecimento específica que compartilham gêneros discursivos particulares para mediar suas atividades de trabalho. Nos encontros, exigiu-se construções enunciativas constituídas sob o princípio da pluralidade, da diversidade das linguagens envolvidas, que exige a compreensão das seguintes linguagens:

Linguagem Química: Os profissionais desta área de conhecimento utilizam representações químicas como forma de comunicação entre eles. Um fenômeno pode ser representado por fórmulas e equações químicas nos seguintes níveis: macroscópico, submicroscópico e simbólico. As representações químicas utilizadas para comunicação entre os profissionais químicos são metáforas, modelos ou constructos teóricos da interpretação química da natureza e da realidade (GIORDAN, 2008, p. 179).

Linguagem da Informática: O gênero discursivo dos profissionais da informática é incorporado, de forma crescente, no cotidiano dos cidadãos em um contexto de mundo globalizado. Os atos de informar, comunicar, acessar, produzir, manipular e armazenar informações na contemporaneidade exige, de seus cidadãos, a utilização de um gênero discursivo específico em uma sociedade em crescente transformação devido ao uso de Tecnologias Digitais.

Linguagem Educacional: $O$ gênero discursivo dos educadores reflete o período de formação do profissional. Provavelmente aqueles cuja formação foi nas décadas de 70 a 80 refletem um discurso tecnicista (PEREIRA et al, 2006, p.16; RICHIT, 2010, p.40); aqueles formados nas décadas de 80 a 90 demonstram um discurso compromissado com a transformação da realidade social (PEREIRA et al, 2006, p.27; RICHIT, 2010, p.40) e, finalmente, aqueles formados após a década de 90 em diante, tendem para um gênero discursivo que ressalta a importância do professor reflexivo, daquele que pensa-na-ação (RICHIT, 2010, p.41; GARRIDO, 2006, p.619). Cabe ressaltar que, devido ao fato das interlocutoras serem doutorandas em Educação, ambas privilegiam modelos de formação nos quais os professores são valorizados como sujeitos criativos e produtores sobre a prática docente.

O terceiro princípio, da historicidade, O SA circunscrito só pode ser entendido no período de constituição e de transformação em âmbito geral da contemporaneidade. Observa-se as crescentes transformações na sociedade, especialmente a partir do uso das dispositivos móveis nos mais variados domínios que envolvem o cotidiano de seus cidadãos. É perceptível que não há como fugir de uma realidade de uso das tecnologias móveis no cotidiano e, inevitavelmente, na Educação. As tecnologias digitais e, em especial, os dispositivos móveis, trazem um potencial de transformação nas formas de se construir o conhecimento e, consequentemente, de ensinar e aprender. O uso dos dispositivos móveis contribuem para o (re)pensar da prática pedagógica. O professor precisa estabelecer conexões e integração entre seus saberes e os dos alunos e, também, que na formação de professores a utilização de tecnologias é muito importante para estimulação de outras formas de fazer docente.

A produção/construção de atividades pedagógicas mediadas por dispositivos móveis para o ensino de Química, num trabalho colaborativo entre as pesquisadoras, é uma atividade em particular. Porém, é possível argumentar que a produção/construção destas 
atividades é uma produção de material de aula, ainda que parcial dentro do currículo do curso de Licenciatura em Química. Mas é preciso considerar que o professor lida com o ensino, ou seja, o trabalho do professor é a produção de material de aula, pois se entende que o ensino se concretiza na aprendizagem.

As tensões e contradições como fontes de mudança e desenvolvimento compõem o quarto princípio. $O$ emprego dos dispositivos móveis no ensino de conhecimentos químicos ocasionou vários momentos de tensões decorrente da incorporação deste novo "artefato", exigindo estudo, negociação e modificação de práticas educativas com esta inovação tecnológica junto aos discentes do curso de licenciatura química. A modalidade m-learning não pode ser tratada apenas como uma questão de mudança metodológica, mas implica na compreensão da história e, consequentemente, na formação das professoras envolvidas nesta pesquisa. Ambas são doutorandas em Educação, cuja formação e atuação profissional como professoras universitárias, foram e são baseadas na modalidade presencial. Isto implica que, ao propor o emprego da mobilidade no processo de ensino-aprendizagem, resulta em grandes mudanças em suas práxis pedagógicas. Foram elaboradas e executadas duas atividades de m-learning junto aos discentes (professores em formação inicial) do curso de licenciatura.

O quinto princípio considera as transformações expansivas, ou seja, aquelas que são fruto das tensões propiciando um ciclo de expansão e desenvolvimento. O ciclo expansivo de desenvolvimento da Formadora de Professores é considerado vital nesta pesquisa, uma vez que ela se defrontou com tensões, que por sua vez, geraram conflitos, que ao serem solucionados proporcionaram seu desenvolvimento. Momentos em que discordou da pesquisadora na utilização de recursos (software, aplicativos, estratégias); ao perceber a dificuldade de digitação na entrada de fórmulas químicas no teclado virtual; na possível substituição do caderno de capa dura (utilizado pelos discentes nas aulas de estágio nas escolas) por tablets para registrar momentos considerados importantes para reflexão (foto/vídeo/textos); a falta de dispositivos móveis para realização das atividades; a falta de rede sem fio (wi-fi) na universidade; entre outros.

Engeström (1999, p.33) considera que o ciclo de desenvolvimento de processo com os movimentos de internalização e externalização nos SA. Engeström (1999) discute o processo de interiorização- exteriorização em todos os níveis da atividade, relacionando à "interiorização à reprodução da cultura e a exteriorização à criação de artefatos que podem ser usados para transformar a cultura" (DANIELS, 1999, p.62).

O ciclo expansivo da formadora de professores resultou em produção de artefatos que indicam o seu desenvolvimento intrapessoal. Para melhor compreensão da sua evolução, ele foi dividido em três ciclos: participação nos encontros, elaboração e aplicação das atividades pedagógicas e elaboração de artigos científicos. A participação nos encontros foi importante pois exigiu uma apropriação reflexiva de ferramentas culturais existentes para solucionar as contradições internas. Na medida em que o ciclo avançou, a externalização passou a dominar propiciando a realização das atividades pedagógicas com os discentes do curso de licenciatura e também a elaboração dos artigos científicos publicados (JACON et al, 2013, 2014a, 2014b). Ao elaborar atividades e artigos científicos, a professora formadora realizou o movimento do abstrato ao concreto, ou seja, concretizou/materializou uma totalidade de conhecimentos que construiu por meio da participação nos encontros. 


\section{Considerações}

A Teoria de Sistema de Atividade possibilitou uma análise detalhada do processo de evolução e desenvolvimento na elaboração de atividades pedagógicas com o uso de dispositivos móveis e, em especial, do ciclo expansivo de desenvolvimento da formadora de professores de química durante este percurso.

Dada a natureza móvel dos dispositivos, o uso de tablets e de smartphones possibilitam mudanças transformadoras nas práticas de ensino aprendizagem. No entanto, para usufruir desta mobilidade é preciso perceber que os professores necessitam de apoio à longo prazo para experimentar o emprego da tecnologia em sua prática escolar para utilizá-la fora da sala de aula (ambientes informais ou não formais). E este apoio deve começar nas licenciaturas junto aos seus formadores para criar e elaborar projetos com esta finalidade. Uma base de conhecimentos sobre espaços de aprendizagem informais poderá incluir novos cenários que extravasem o ensino na sala de aula, e esta iniciativa pode ser resultado da participação e colaboração interdisciplinar entre pesquisadores de Informática e Educação e Formadores de Professores. 


\section{Referências}

DANIELS, Harry. Vygotsky e a pedagogia. Ed.Loyola. São Paulo, Brasil, 2003. 246p.

ENGESTRÖM, Y. Activity theory and individual and social transformations. In: ENGESTRÖM, Y.; MIETTINEN, R.; PUNAMAKI, R. (Orgs.). Perspectives on Activity Theory. Cambridge: Cambridge University Press, 1999

CAMILLO, Juliano; MATTOS, Cristiano Rodrigues de. A experimentação no ensino de ciências: reflexões a partir da Teoria da Atividade. (cap.5) In: MAGALHÃES JUNIOR, C.A.de O.; LORENCINI JUNIOR, A.; CORAZZA, M.J. (org.) Ensino de Ciências: Múltiplas perspectivas, diferentes olhares. Curitiba: CRV Editora, 2014, p.123-154. ISBN:978-85-444-0028-9

GARRIDO, Elsa; BRZEZINSKI, Iria. A pesquisa na formação de professores. In: Formação de educadores: artes e técnicas - ciências e políticas. Org. Raquel L.L. Barbosa. Editora Unesp. São Paulo, 2006. pp.605-616

JACON, Liliane S.C.;MARTINES, E.A.L.de M.; OLIVEIRA, A.C.G.de; MELLO, I.C.de. Educação \& Tecnologia: Reflexões sobre a incorporação de tecnologias móveis na Educação. In: Revista da Rede Amazônica de Educação em Ciências e Matemática - Reamec Cuiabá/MT, n.01, set/2013, p.88-101, ISSN 2318-6674. Disponível em <http://www.ufmt.br/ufmt/unidade/index.php/secao/site /5394/ppgecem> Acesso em nov.2013.

JACON, Liliane S.C.; MELLO, I.C.de; OLIVEIRA, A.C.G.de. Aprendizagem com Mobilidade no ensino de conhecimentos químicos: Reflexões de uma pesquisa realizada com professores em formação inicial. Revista Educação a Distância e Práticas Educativas Comunicacionais e Interculturais EDaPECI, Universidade Federal de Sergipe em Parceria com a Universidade Federal de Alagoas (UFS), v.14, n.1, p.235-248, 2014. Disponível em: <http://www.seer.ufs.br/index.php/edapeci >. ISSN 2176-171X

JACON, Liliane S.C.;MARTINES, E.A.L.de M.; OLIVEIRA, A.C.G.de; MELLO. Os formadores de professores e o desafio em potencializar o aprendizado em ciências químicas com a incorporação dos dispositivos móveis In: Revista IENCI Investigações em Ensino de Ciências. Porto Alegre/RS, V.19(1) pp.77-89 2014. UFRGS. Disponível em http://www.if.ufrgs.br/ienci/artigos/Artigo_ID356 /v19_n1_a2014.pdf ISSN 1518-8795

LEONTIEV, A. N. Uma contribuição à teoria de desenvolvimento da psique infantil. In: VIGOTSKI, L. S.; LURIA, A. R.; LEONTIEV, A. N. Linguagem, desenvo/vimento e aprendizagem. São Paulo: Ícone, 2001. p. 59-83.

PEREIRA, Júlio Emílio Diniz. Debates e pesquisas no Brasil sobre formação docente. In: Pereira, Júlio Emílio Diniz. Formação de professores: Pesquisas, representações e poder. 2. ed. Belo Horizonte: Autêntica, 2006. p. 15 - 52.

RICHIT, Adriana. Apropriação do Conhecimento Pedagógico-Tecnológico em Matemática e a Formação Continuada de Professores. Tese de doutorado. Unesp, Rio Claro, SP. 2010.

SACCOL, Amarolinda; SCHLEMMER, Eliane; BARBOSA, Jorge. M-learning e U-learning: novas perspectivas da aprendizagem móvel e ubíqua. São Paulo, edt. Pearson Prentice Hall, 2011. 162p. 